\title{
Fluorophore-Doped Core-Multishell Spherical Plasmonic Nanocavities: Resonant Energy Transfer toward a Loss Compensation
}

\author{
Bo Peng, ${ }^{\dagger}$ Qing Zhang, ${ }^{\dagger}$ Xinfeng Liu, ${ }^{\dagger}$ Yun $\mathrm{Ji}^{+}{ }^{\dagger}$ Hilmi Volkan Demir, ${ }^{+, \neq, \S}$ Cheng Hon Alfred Huan, ${ }^{\dagger}$ \\ Tze Chien Sum, ${ }^{\dagger}$ and Qihua Xiong ${ }^{t, \neq, *}$ \\ ${ }^{\dagger}$ Division of Physics and Applied Physics, School of Physical and Mathematical Sciences, Nanyang Technological University, Singapore 637371, \\ ${ }^{\ddagger}$ Division of Microelectronics, School of Electrical and Electronic Engineering, Nanyang Technological University, Singapore 639798, and ${ }^{\S}$ Department of Electrical \\ and Electronics Engineering, Department of Physics, UNAM-National Nanotechnology Research Center, Bilkent University, Bilkent Ankara, Turkey 06800
}

$\mathrm{P}$ lasmonics, which deals with collective electron oscillations in metallic nanostructures, offers the opportunity to enable routing and active manipulation of light at the subwavelength scale and promises a family of exciting applications such as invisibility, optical data processing, and quantum information. ${ }^{1-4}$ Nevertheless, metallic Joule loss, an intrinsic feature of metalbased waveguides supporting the surface plasmon propagation, hampers the achievement of excellent optical properties for nanophotonics applications. Therefore, the losses impose key challenges in fundamental plasmonic research and relevant technological development. Recently, there is a tremendous interest in the plasmon loss compensation by introducing gain media. ${ }^{5-7}$ Optically pumped gain media (dye molecules, quantum dots, etc.) have been incorporated into the adjacent dielectric to compensate the losses that dampen the coupled oscillations of electrons and light. $^{8-10}$ Pusch and Wuestner et al. theoretically demonstrated the possibility of compensating the loss by gain in a negative refractive index double-fishnet metamaterial with an embedded laser dye on the basis of a fullvectorial three-dimensional Maxwell-Bloch approach. ${ }^{11-13}$ Zheludev et al. experimentally demonstrated a Joule loss compensation using optically pumped semiconductor quantum dots hybridized to plasmonic nanostructures, leading to a multifold intensity increasing and narrowing of their photoluminescence (PL) spectrum due to Purcell effect. ${ }^{14,15}$ Meanwhile, gain compensation opens a promising avenue to achieve surface plasmon laser, so-called SPASERs. ${ }^{16-19}$ The results from these previous experiments suggest

\section{ABSTRACT}
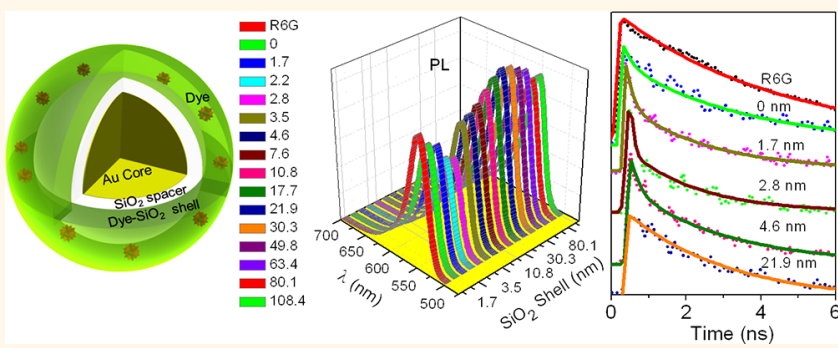

Plasmonics exhibits the potential to break the diffraction limit and bridge the gap between electronics and photonics by routing and manipulating light at the nanoscale. However, the inherent and strong energy dissipation present in metals, especially in the near-infrared and visible wavelength ranges, significantly hampers the applications in nanophotonics. Therefore, it is a major challenge to mitigate the losses. One way to compensate the losses is to incorporate gain media into plasmonics. Here, we experimentally show that the incorporation of gain material into a local surface plasmonic system (Au/silica/silica dye core-multishell nanopartides) leads to a resonant energy transfer from the gain media to the plasmon. The optimized conditions for the largest loss compensation are reported. Both the coupling distance and the spectral overlap are the key factors to determine the resulting energy transfer. The interplay of these factors leads to a non-monotonous photoluminescence dependence as a function of the silica spacer shell thidkness. Nonradiative transfer rate is increased by more than 3 orders of magnitude at the resonant condition, which is key evidence of the strongest coupling ocauming between the plasmon and the gain material.

KEYWORDS: core-multishell · plasmonic nanocavities · loss compensation · resonant energy transfer $\cdot$ nonradiative rate

the occurrence of the loss compensation, where the emission energy is coupled from the excited state of an adjacent fluorophore to the plasmon resonance through near-field interactions. $^{20-25}$

Recently, many studies have been devoted to the interactions between fluorophores and the plasmon resonances. The fluorescence intensity quenching or
* Address correspondence to qihua@ntu.edu.sg.

Received for review April 17, 2012 and accepted June 12, 2012.

Published online June 12, 2012 $10.1021 / \mathrm{nn} 301716 \mathrm{q}$

(c) 2012 American Chemical Society 

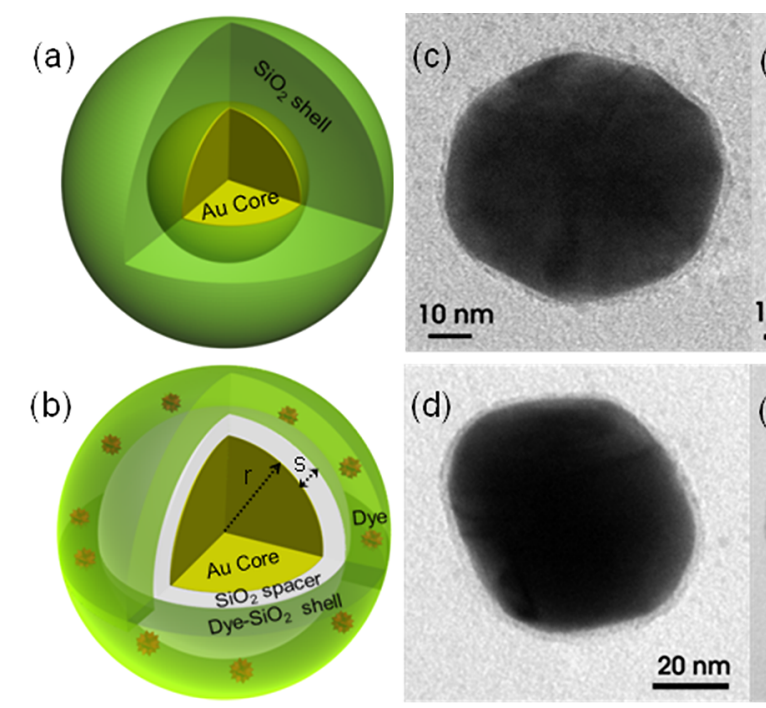

(e)
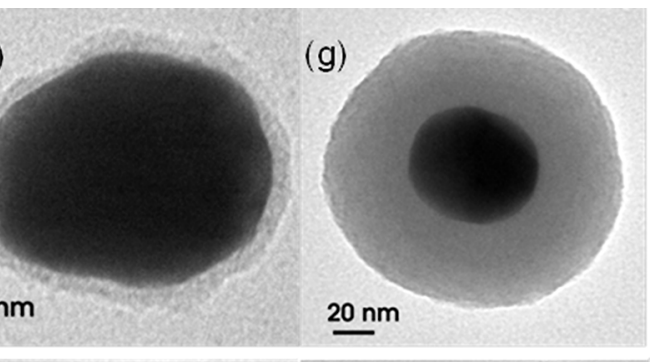

(f)

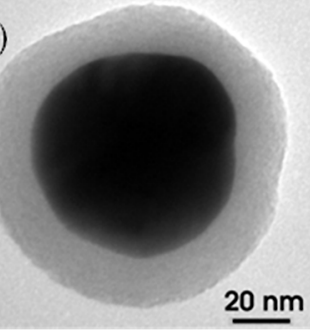

(h)

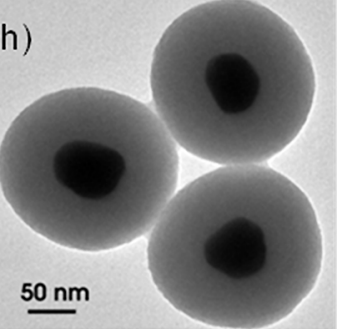

Figure 1. Core-shell/multishell plasmonic nanocavities. (a) Schematic diagram of Au(yellow)/silica(green) core-shell nanostructures. (b) Schematic diagram of Au/silica/dye silica core-multishell nanostructures. Middle silica shell (shown in white) is used as a spacer to control the distance between Au particles and dye; $r$ is the radius of Au particles, and $s$ is the thickness of the spacer. Dye molecules (shown as red stars) are dispersed in the outermost silica shell (shown as green shell). (c-g) TEM images of $\mathrm{Au} /$ silica core-shell structures with different shell thickness: (c) $1.7 \mathrm{~nm}$, (d) $2.8 \mathrm{~nm}$, (e) $4.6 \mathrm{~nm}$, (f) $17.7 \mathrm{~nm}$, (g) $39.5 \mathrm{~nm}$. (h) TEM images of Au/silica/dye (Rhodamine 6G) silica core-multishell nanostructures. Silica spacer thickness is $2.8 \mathrm{~nm}$, and the thickness of R6G silica layer is $54.2 \mathrm{~nm}$.

enhancement as a function of the distance between the fluorophores and the metal nanoparticles is a fundamental question to be addressed. ${ }^{26-28}$ There have been a few attempts to use polyelectrolyte or DNA linkers to control the distance; however, a rigid dielectric spacer layer is more advantageous to accurately control the spacer thickness and investigate the energy transfer in the sub- $5 \mathrm{~nm}$ regime, which is critical to address the recent debate on the distance of quenching and enhancement maximum. ${ }^{29-32}$ The systematic studies of the variation in fluorescence intensity as a function of the distance between gain materials and plasmon, which is an essential step toward understanding the loss compensation, still remain elusive. In addition to this distance dependence, the local field enhancement surrounding metal nanostructures is strongly wavelength-dependent. Therefore, loss compensation will also depend on the spectral overlap between the gain media and plasmon.

The aim of our work is to explore the optimized conditions for loss compensation in plasmon with gain media. We systematically investigate the distance dependence and spectral overlap effect of the energy transfer between plasmon and fluorophores based on a Au core, a rigid silica spacer shell, and rhodamine $6 \mathrm{G}$ dyes (R6G) or fluorescein isothiocyanate (FITC) which are embedded into another silica shell. Our results show that the energy transfers from R6G to plasmon when the silica spacer is smaller than $7.6 \mathrm{~nm}$. On the contrary, the energy transfer direction is from plasmon to R6G above $7.6 \mathrm{~nm}$. The PL intensity of R6G exhibits a minimum when the spacer is $2.8 \mathrm{~nm}$ and a maximum at $21.9 \mathrm{~nm}$. Therefore, we suggest the optimized distance for the energy transfer from R6G to plasmon is $2.8 \mathrm{~nm}$, which indicates the largest loss compensation. Time-resolved fluorescence spectroscopy shows that the lifetime of R6G also exhibits a minimum at $2.8 \mathrm{~nm}$ spacer. The nonradiative rate increases by more than 3 orders of magnitude, and the quantum efficiency also reduces down to $0.02 \%$ from $95 \%$, which further confirms the strongest interaction between plasmon and R6G.

\section{RESULTS AND DISCUSSION}

Surface Plasmon Electronic Oscillations in Au/Silica CoreShell Structures. We designed and constructed a coremultishell plasmonic nanocavity system consisting of a Au core with a diameter of about $60 \mathrm{~nm}$, surrounded by a silica shell as a spacer which is coated with another silica shell with dye molecules embedded (Figure 1a,b). The thickness of the spacer silica shell can be tuned from 1.7 to $108.4 \mathrm{~nm}$ by solution chemistry, obtained from statistical analysis of the electron micrographs. In such plasmonic nanocavities, Au core underpins the plasmonic modes, the outer silica shell containing the organic dye molecules (shown as red stars) provides a gain media, and the middle silica shell (shown in white) functions as a spacer to tune the distance between the plasmon and gain. It is important to note that the spacer can also fine-tune the plasmonic bands. Figure $1 c-f$ shows the TEM images of $\mathrm{Au} / \mathrm{silica}$ core-shell structures, and the thickness of silica shell is $1.7 \pm 0.2,2.8 \pm 0.4,4.6 \pm 0.8,17.7 \pm 0.6$, and $39.5 \pm 0.9 \mathrm{~nm}$, respectively. The standard deviation is obtained by statistical analysis. Figure $1 \mathrm{~h}$ shows the Au/silica spacer/dye silica multilayer hybrid coremultishell structures, in which the embedded dye 

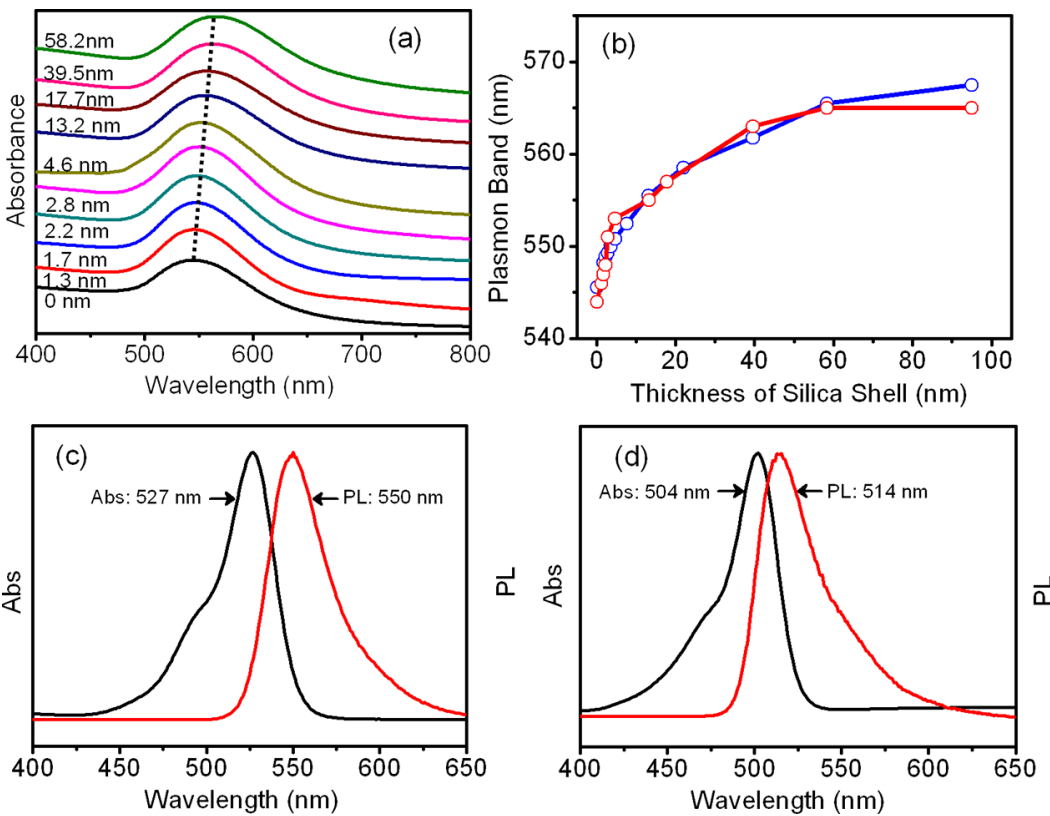

Figure 2. Spectroscopy characterizations. (a) Normalized absorption spectra of aqueous solutions of Au nanoparticles and $\mathrm{Au} / \mathrm{silica}$ core-shell nanostructures with different shell thickness; $0 \mathrm{~nm}$ represents the bare Au nanoparticles. (b) Plasmon band position as a function of the silica shell thickness obtained in experimental data (red open circles + line), and FDTD simulation (blue open circles +line). (c,d) Normalized absorption and photoluminescence spectra of Rhodamine 6G (R6G) and FITC-APS conjugate. From (a) and (c), we know that R6G emission is in resonance with Au nanoparticles with a $2.8 \mathrm{~nm}$ silica shell.

molecule is R6G, the spacer shell is $2.8 \mathrm{~nm}$, and the dye silica shell is $54.2 \pm 0.5 \mathrm{~nm}$.

For non-interacting metallic particles in a nonabsorbing medium, their extinction coefficient is given by Mie theory, which describes a summation over all electric and magnetic multipolar oscillations contributing to the absorption and scattering of the interacting electromagnetic field. The extinction coefficient of a particle is given by ${ }^{33}$

$$
C_{\text {ext }}=\frac{18 \pi V \varepsilon_{\mathrm{m}}^{3 / 2} \varepsilon_{2}}{\lambda\left(\left(\varepsilon_{1}+2 \varepsilon_{\mathrm{m}}\right)^{2}+\varepsilon_{2}^{2}\right)}
$$

where $\varepsilon_{\mathrm{m}}$ is the real part of the dielectric function of the surrounding medium, and $\varepsilon_{1}, \varepsilon_{2}$ represent the real and imaginary parts of the dielectric function of the material, respectively $\left(\widetilde{\varepsilon}_{\text {core }}=\varepsilon_{1}+i \varepsilon_{2}\right) . V$ is the molar volume of the material constituting the particles. The absorbance of a colloidal solution containing $N$ particles in an optical cell with a path length $L$ is given by $A=C_{\text {ext }} N L /$ In $10 .{ }^{34}$ This theory has been extended to ellipsoids and multilayer particles. ${ }^{35}$ The exact position of the plasmon absorption band is extremely sensitive to the particle size and shape, and the dielectric constant of the surrounding medium. For core-shell structures, the extinction cross section is given by ${ }^{36}$

$$
\begin{gathered}
C_{\text {ext }}=4 \pi r^{2} k^{*} \operatorname{Im} \\
\left\{\frac{\left(\varepsilon_{\text {shell }}-\varepsilon_{m}\right)\left(\varepsilon_{\text {shell }}-2 \widetilde{\varepsilon}_{\text {core }}\right)+(1-h)\left(\widetilde{\varepsilon}_{\text {core }}-\varepsilon_{\text {shell }}\right)\left(\varepsilon_{m}+2 \varepsilon_{\text {shell }}\right)}{\left(\varepsilon_{\text {shell }}+2 \varepsilon_{m}\right)\left(\tilde{\varepsilon}_{\text {core }}+2 \varepsilon_{\text {shell }}\right)+(1-h)\left(2 \varepsilon_{\text {shell }}-2 \varepsilon_{m}\right)\left(\widetilde{\varepsilon}_{\text {core }}-\varepsilon_{\text {shell }}\right)}\right\}
\end{gathered}
$$

where $\widetilde{\varepsilon}_{\text {core }}$ is the complex dielectric functions of the core and $\varepsilon_{\text {shell }}$ is the real part of shell material dielectric function; $h$ is the volume fraction of the shell layer, $h=1-r^{3} /(r+s)^{3}$, where $r$ is the radius of metallic particle and $s$ is the thickness of shell. The resonance condition for the plasmon absorption is roughly fulfilled when the denominator is equal to $0^{37}$

$$
\varepsilon_{1}=-\frac{2 \varepsilon_{\text {shell }}\left[h \varepsilon_{\text {shell }}+\varepsilon_{\mathrm{m}}(3-h)\right]}{\varepsilon_{\text {shell }}(3-2 h)+2 h \varepsilon_{\mathrm{m}}}
$$

According to Drude model, the real $\left(\varepsilon_{1}\right)$ and imaginary $\left(\varepsilon_{2}\right)$ parts of the dielectric function are given by $\varepsilon_{1}=\varepsilon_{\infty}-\omega_{\mathrm{p}}^{2}\left(\omega^{2}+\omega_{\mathrm{d}}^{2}\right)$ and $\varepsilon_{2}=\omega_{\mathrm{p}}^{2} \omega_{\mathrm{d}} / \omega\left(\omega^{2}+\omega_{\mathrm{d}}^{2}\right)$, where $\varepsilon_{\infty}$ is the high-frequency dielectric constant of gold and $\omega_{\mathrm{p}}$ is the bulk plasma frequency expressed in terms of the free electron density $n$, the electron charge $e$, the vacuum permittivity $\varepsilon_{0}$, and the electron effective mass $m_{\mathrm{eff}}, \omega_{\mathrm{p}}^{2}=n e^{2} / m_{\mathrm{eff}} \varepsilon_{0} ; \lambda_{\mathrm{p}}$ is the bulk plasma wavelength of metal, $\lambda_{\mathrm{p}}=2 \pi c / \omega_{\mathrm{p}} . \omega_{\mathrm{d}}$ is the relaxation or damping frequency. Therefore, the band position for core-shell structures should obey

$$
\frac{\lambda^{2}}{\lambda_{\mathrm{p}}^{2}}=\varepsilon_{\infty}+\frac{2(1-h) \varepsilon_{\text {shell }}\left(\varepsilon_{\text {shell }}-\varepsilon_{\mathrm{m}}\right)+6 \varepsilon_{\text {shell }} \varepsilon_{\mathrm{m}}}{3 \varepsilon_{\text {shell }}-2(1-h)\left(\varepsilon_{\text {shell }}-\varepsilon_{\mathrm{m}}\right)}
$$

From eq 4, we find that the plasmon absorption band is red-shifted as the thickness of the shells increases when $\varepsilon_{\text {shell }}>\varepsilon_{\mathrm{m}}$.

Figure $2 \mathrm{a}$ shows the UV-vis absorption spectra of $\mathrm{Au} / \mathrm{silica}$ core-shell structures with different shell thickness ( $0 \mathrm{~nm}$ corresponds to bare Au nanoparticles), 

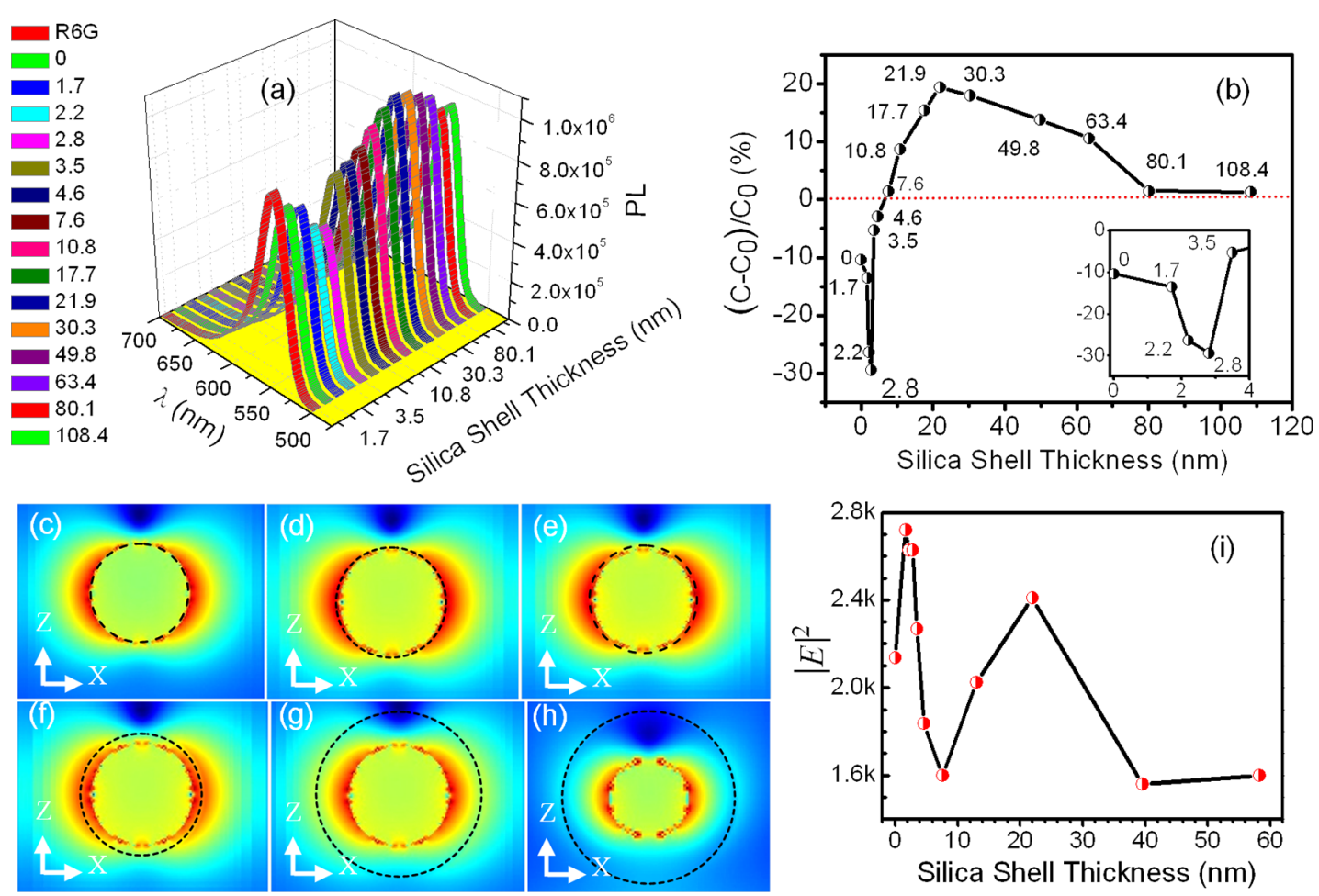

Figure 3. (a) Photoluminescence spectra of aqueous solution of R6G containing Au/silica core-shell nanostructures with different shell thickness. Non-monotonous behavior is noticed. (b) Plot of the variation $\left(C-C_{0}\right) / C_{0}$ of the PL intensity as a function of the silica shell thickness extracted from (a); the inset is the zoomed-in view around $2.8 \mathrm{~nm}$. Local electric field contours for Au/silica core-shell structures with different shell thickness: (c) $0 \mathrm{~nm}$ (bare Au nanoparticles), (d) $1.7 \mathrm{~nm}$, (e) $2.8 \mathrm{~nm}$, (f) $7.6 \mathrm{~nm},(\mathrm{~g}) 21.9 \mathrm{~nm}$, (h) $39.5 \mathrm{~nm}$. The dotted circles indicate the edge of silica shell. (i) Electric field enhancement $|E|^{2}$ as a function of the silica spacer thickness. The wavelength of local field intensity distribution corresponds to the plasmon band position of $\mathrm{Au} / \mathrm{silica}$ core-shell structures, which are shown in Figure $\mathbf{2 b}$.

dispersed into water solutions. Au colloids show a very intense surface plasmon absorption band around $544 \mathrm{~nm}$, which red shifts systematically due to the fact that the dielectric constant of the silica shell $\left(\varepsilon_{\text {shell }}=2.12\right)$ is larger than that of the solvent $\left(\mathrm{H}_{2} \mathrm{O}, \varepsilon_{\mathrm{m}}=1.78\right) .{ }^{37}$ As the shell thickness increases, the surface plasmon resonance band red shifts from $544 \mathrm{~nm}$ for bare nanoparticles to $565 \mathrm{~nm}$ for $95 \mathrm{~nm}$ shell nanoparticles, which is in agreement with eq 4 . The experimental surface plasmon resonance band position versus the shell thickness is plotted in Figure $2 b$ (red open circles + line). We also calculated the surface plasmon resonance position by the finite-difference time-domain method (FDTD), as depicted in Figure 2b (blue open circles + line), showing good agreement with experimental data. ${ }^{38}$ In order to investigate the coupling between surface plasmon and gain medium, R6G and FITC dye molecules are used as the gain materials. Their peak positions of the absorption and $\mathrm{PL}$ maximum are 527 and $550 \mathrm{~nm}$ for R6G (Figure 2c) and 502 and $514 \mathrm{~nm}$ for FITC-(3-aminopropyl)trimethoxysilane (FITC-APS) conjugate (Figure 2d), respectively. Compared with Figure $2 \mathrm{a}, \mathrm{c}$, we find that the surface plasmon band of Au/silica core-shell structures is in resonance with the emission of R6G molecules when the shell thickness is $\sim 2.8 \mathrm{~nm}$ with a resonance of $\sim 551 \mathrm{~nm}$, where the FITC emission energy is higher than the surface plasmon resonance band (Figure 2a,d).

Spacer Distance Dependence of Energy Transfer between Plasmon and Gain Medium. On the basis of Au/silica core-shell structures, we systematically investigate the distance dependence of energy transfer between plasmon and gain medium (R6G) by evaluating the enhancing or quenching effect on dye molecule emission in the presence of plasmonic nanoparticles. Silica shell is used as a spacer to control the distance between $\mathrm{Au}$ and R6G, while keeping the concentrations of the particles and dye molecules constant. Figure 3a shows the room-temperature PL spectra of the aqueous solution of R6G (excited at $480 \mathrm{~nm}$ ) containing Au/silica core-shell structures with different shell thickness. Presumably, only those R6G molecules getting in contact with plasmonic nanoparticles will be significantly affected in terms of their quantum yield of luminescence. A pronounced silica shell thickness dependence is spotted from Figure 3a. The PL intensities are extracted and plotted versus shell thickness in Figure $3 \mathrm{~b}$, where $C$ and $C_{0}$ are the PL intensities of R6G with and without $\mathrm{Au} / \mathrm{silica}$ core-shell particles, respectively. When the spacer is $7.6 \pm 0.9 \mathrm{~nm}$, the PL intensity is comparable to that of only R6G in the aqueous solution. Below $7.6 \mathrm{~nm}$, the PL is quenched, while the $\mathrm{PL}$ is enhanced above $7.6 \mathrm{~nm}$. When the shell is thicker 
than $\sim 80 \mathrm{~nm}$, the surface plasmon has barely any effect on the PL signal of R6G within the experimental error, which indicates that the energy transfer between plasmon and gain occurs only when the spacer is smaller than $\sim 80 \mathrm{~nm}$. Our results are consistent with previous studies. ${ }^{32,39-41}$ Two interesting phenomena are observed: the PL exhibits a local minimum (strongest quenching) when the spacer is $2.8 \mathrm{~nm}$ and a local maximum (strongest enhancement) at $21.9 \mathrm{~nm}$, which are totally different from the previous experimental works. ${ }^{39,41,42}$

Theoretically, the optical quenching of donor fluorophores by proximal Au NPs has been formulated. ${ }^{43}$ Focusing on the dye-Au nanoparticle system, Strouse and co-workers proposed to model the system as a point dipole interacting with an infinite metal surface and to integrate the Förster expression over the twodimensional surface, which leads to a $1 / d^{4}$ dependence of the energy transfer rate, where $d$ is the separation distance between $\mathrm{Au}$ particle and dye molecules. ${ }^{29}$ Carminati et al. further derived the expressions for the distance dependence by using Green function formalism, and the rate of energy transfer, $\kappa_{\mathrm{T}}$, can be given by ${ }^{30,44}$

$$
\kappa_{\mathrm{T}}=\frac{1+\frac{1}{6}\left(2 \pi n d / \lambda_{\mathrm{d}}\right)^{2}+\frac{1}{6}\left(2 \pi n d / \lambda_{\mathrm{d}}\right)^{4}}{\tau_{\mathrm{D}}\left(d / R_{0(\text { FRET })}\right)^{6}}
$$

where $R_{0 \text { (FRET) }}$ is the Förster separation distance corresponding to $50 \%$ efficiency. However, only at large separation distances $(>30 \mathrm{~nm})$, the higher order corrections from $\left(d / \lambda_{\mathrm{d}}\right)^{2}$ and $\left(d / \lambda_{\mathrm{d}}\right)^{4}$ are expected. At a small distance, the rate of energy transfer is equivalent to the Förster dipole-dipole interaction model, which can be given by ${ }^{45}$

$$
\kappa_{\mathrm{T}}=\frac{1}{\tau_{\mathrm{D}}\left(d / R_{0(\mathrm{FRET})}\right)^{6}}
$$

where $\tau_{\mathrm{D}}$ is the lifetime of the donor in the absence of acceptor. $R_{\text {(FRET) }}^{6}=8.79 \times 10^{-5}\left(\kappa^{2} n^{-4} Q_{\mathrm{d}}\right)$ ), in the unit of $\AA^{6}$, ${ }^{45}$ where $n$ is the refractive index of the medium and $\lambda_{\mathrm{d}}$ is the donor emission wavelength. $Q_{\mathrm{d}}$ is the quantum yield of donor in the absence of Au particles, which is 0.95 for R6G. ${ }^{46}$ The $\kappa^{2}$ is the dipole orientation factor, which is 2 for Au particle acceptors. ${ }^{30} \mathrm{~J}$ is the spectral overlap integral between the normalized donor emission and the acceptor absorption. ${ }^{45}$ Therefore, the larger the spectral overlap is, the faster the energy transfer and the stronger local quenching is. We simulated the local electric field enhancement by a FDTD method. Figure $3 c-h$ shows the calculated local field intensities distributed in the $x-z$ plane for bare $A u$ nanoparticles and $\mathrm{Au} /$ silica core-shell structures with $1.7,2.8,7.6,21.9$, and $39.5 \mathrm{~nm}$ silica shells, respectively. Figure 3i plots the square of maximum electric field $|E|^{2}$, as a function of the spacer thickness. $|E|^{2}$ increases to a maximum when the thickness of the silica shell is
1.7-2.8 $\mathrm{nm}$ and then monotonously drops to a minimum at $\sim 7.6 \mathrm{~nm}$. A second maximum at $\sim 21.9 \mathrm{~nm}$ is observed beyond which the $|E|^{2}$ decreases. When the silica shell is more than $40 \mathrm{~nm},|E|^{2}$ shows a very weak dependence on the spacer thickness. Generally speaking, two competing factors determine the overall quenching and enhancement of the luminescence intensity: increased absorption and emission due to the coupling of the radiative mode of the transition dipole with the surface plasmon, and the nonradiative energy transfer from the excited dipole to the metal. ${ }^{47}$ Therefore, we suggest that nonradiative energy transfer predominates at short distances $(<7.6 \mathrm{~nm})$. Both the small distance and the large spectral overlap between PL and the plasmon band are the key factors, which yields a fast nonradiative energy transfer from the excited dipole to the metal. Since the spectral overlap between the surface plasmon band and emission of R6G is the largest at $2.8 \mathrm{~nm}$ spacer, which is in good resonance, the quenching becomes the strongest. In the case of the enhancement of the luminescence intensity at long distances $(>7.6 \mathrm{~nm})$, the excitation rate is enhanced. Because the absorption increases due to the local surface plasmon, $\gamma_{\text {exc }} \propto|p \cdot E|^{2}$, where $p$ is the transition dipole moment of dye molecule. ${ }^{26}$ The radiative decay rate with and without plasmon, $r_{\text {rad }}^{\mathrm{sp}}$ and $r_{\text {rad, }}^{0}$ are related by $|E|^{2}, r_{\text {rad }}^{\text {sp }} \propto$ $|E|^{2} r_{\text {rad. }}^{0}{ }^{48}$ Therefore, the radiative rate is enhanced significantly (Purcell effect); ${ }^{24,44,49-51}$ meanwhile, the nonradiative rate is suppressed in the surface plasmon enhanced system. Therefore, the enhancement factors are large enough that we see an overall increase in PL intensity, which is proportional to $|E|^{2}{ }^{2} 32,47,52$

Gain-Assisted Spherical Gold Plasmon Nanostructures. Time-resolved fluorescence spectroscopy allows us to gain an understanding of the coupling between dye molecules and plasmon nanoparticles, which is critically dependent on the distance between them. This is achieved in a hybrid core-multishell nanostructure consisting of a Au particle core, a silica shell as a spacer, and a R6G dye-doped silica shell as the outer layer (shown in Figure 1b). It is important to note that we also measured the time-resolved PL of a mixture of a R6G solution containing Au/silica core-shell nanostructures. However, this approach shows a negligible difference of the PL decay constants with a control experiment of a R6G aqueous solution without $\mathrm{Au} /$ silica nanostructures. We believe that the fast collision between R6G molecules and Au/silica nanostructures makes it difficult to resolve the plasmon-exciton coupling, if any, by time-resolved PL. In our current core-multishell nanostructure with a dye-doped silica shell, it is possible to bring the gain media with a controlled concentration to a single gold nanoparticle level and precisely control the spacer thickness, both of which are essential for the understanding of the strong 

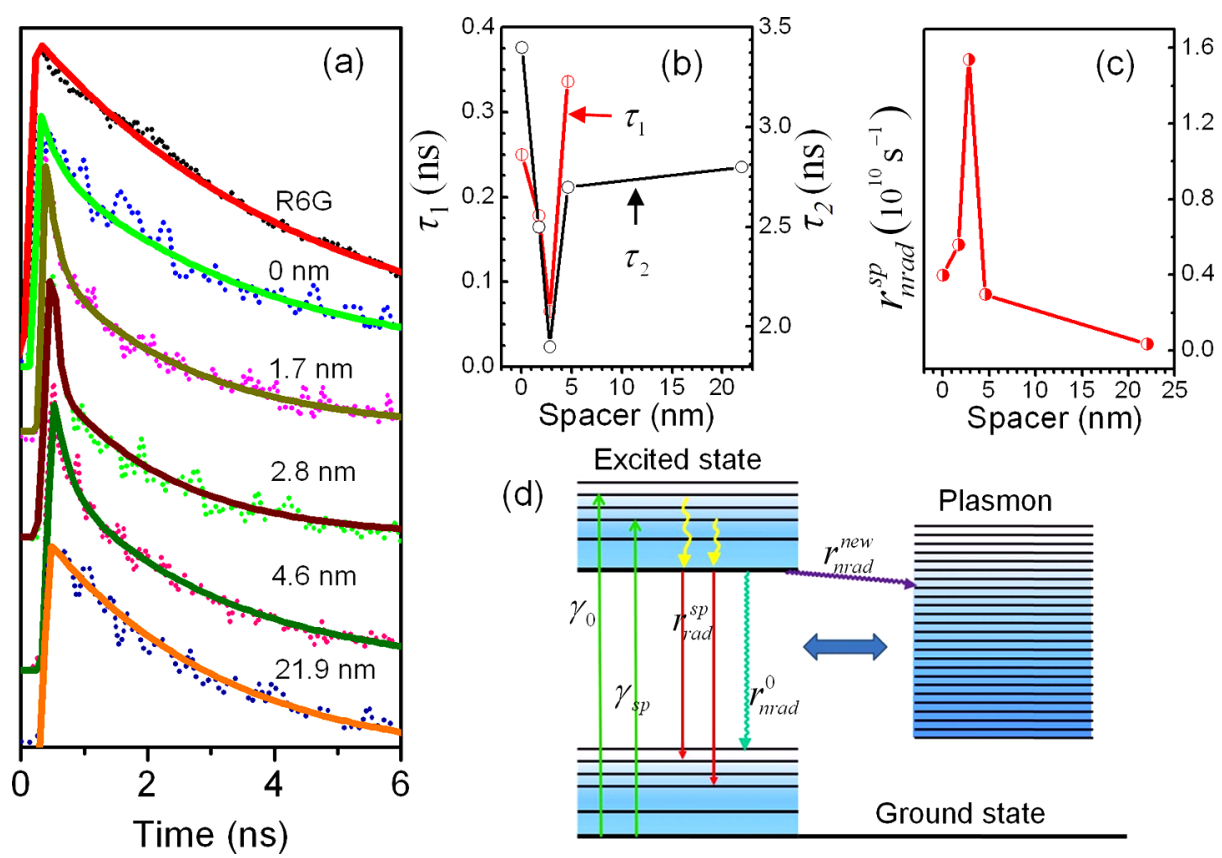

Figure 4. (a) Time-resolved fluorescence spectra of R6G and Au/silica/R6G silica core-multishell structures. The silica spacer thicknesses are $0,1.7,2.8,4.6$, and $21.9 \mathrm{~nm}$, respectively. The solid lines are fits to the data using an exponential decay function, as described in the text. (b) Lifetime as a numerical function of the silica spacer thickness ( $\tau_{1}$ red line, $\tau_{2}$ black line). (c) Nonradiative rate $\left(r_{\text {nrad }}^{\text {sp }}\right)$ as a function of the silica spacer thickness. The unit is $10^{10} \mathrm{~s}^{-1}$. (d) Jablonski diagram for molecular fluorescence excitation and decay on a plasmonic system: direct photon excitation without plasmon $\left(\gamma_{0}\right)$ and enhanced excitation due to plasmon $\left(\gamma_{\mathrm{sp}}\right)$ (green), radiative model with plasmon $\left(r_{\mathrm{rad}}^{\mathrm{sp}}\right.$, red), nonradiative model without plasmon ( $r_{\text {nrad }}^{0}$ cyan), and new decay nonradiative model due to plasmon ( $r_{\text {nrad, }}^{\text {new }}$ violet).

plasmon-exciton resonant coupling toward novel loss compensation in plasmonics.

Figure 4a shows time-resolved PL spectra of R6G and Au/silica/R6G silica core-multishell nanostructures. The silica spacer thicknesses are $0,1.7,2.8,4.6$, and $21.9 \mathrm{~nm}$, respectively. For the R6G aqueous solution, the time-resolved PL decay curve can be well fitted as a single-exponential function shown in Figure $4 a$ (black dots and red line fit), resulting in a lifetime of $\tau_{\mathrm{f}}=4.1 \pm 0.1 \mathrm{~ns}$, which is consistent with previous studies. ${ }^{53}$ For Au/silica/R6G silica nanostructures with the spacer thickness between 0 and $4.6 \mathrm{~nm}$, the PL decay curves of R6G cannot be well fitted by a single-exponential function. Rather, a biexponential decay function gives a better fit, $I(t)=A_{1} \exp \left(-t / \tau_{1}\right)+$ $A_{2} \exp \left(-t / \tau_{2}\right),{ }^{54}$ with two time constants: a fast decay $\left(\tau_{1}\right)$ accompanied by a long-living emission $\left(\tau_{2}\right)$, where $I(t)$ is the PL intensity. However, when the spacer thickness is $21.9 \mathrm{~nm}$, the PL decay can be fitted as a single-exponential function with a time constant of $2.8 \pm 0.1$ ns, which shows a pronounced decrease compared to R6G alone, suggesting an increase of the radiative recombination rate. ${ }^{55}$ Pons et al. and Ray et al. reported that plasmon-induced multiexponential fluorescence decay is due to dipole-metal interactions. ${ }^{30,41}$ Dulkeith et al. found that short- and long-living emission components can be identified in the decay dynamics of the dye/Au nanoparticle system. The long-living emission decay is attributed to dye molecule far away from $\mathrm{Au}$ nanoparticles. ${ }^{56}$ In our case, the thickness of the R6Gdoped silica layer is $54.2 \mathrm{~nm}$. Therefore, we suggest that the long-living emission is related to the fraction of dye molecules in the silica shell, which are located at longer distances from plasmonic Au nanoparticles, thus exhibiting a very weak interaction with the $\mathrm{Au}$ core. The fast decay time is attributed to the fraction of R6G dye decorating the silica shell that experiences the resonant energy transfer process, which is consistent with the opening up of additional relaxation pathways due to the surface plasmon.

Figure $4 b$ shows the decay lifetime as a function of spacer shell thickness in Au/silica/R6G silica coremultishell plasmon nanostructures. The two decay lifetimes are $\left(\tau_{1}\right) 250 \pm 40,178 \pm 10,65 \pm 9$, and $336 \pm$ $16 \mathrm{ps}$ and $\left(\tau_{2}\right) 3.4 \pm 0.1,2.5 \pm 0.1,1.9 \pm 0.1$, and $2.7 \pm$ $0.1 \mathrm{~ns}$ in the case that the silica spacers are $0,1.7,2.8$, and $4.6 \mathrm{~nm}$, respectively. Many previous works have reported the significant decrease of lifetime in dyeplasmon systems coupled via DNA or polyelectrolyte linkage, which can be essentially traced back to an increase of the nonradiative rate leading to fluorescence quenching. ${ }^{30,57,58}$ Ray et al. investigated the distance dependence of the fluorescence lifetime of dye molecules. They found that the lifetime was monotonously increased as the distance between plasmon and fluorophore was increased. ${ }^{41}$ However, in our case, we observe that the lifetime first decreases strikingly to a minimum at $2.8 \mathrm{~nm}$ and then increases monotonously 
when the spacer thickness increases even further. To obtain more quantitative information, we evaluate the fluorescence decay rate using $r_{\mathrm{f}}=1 / \tau_{\mathrm{f}}$. The decay rate has both radiative and nonradiative components and can be expressed as $r_{\mathrm{f}}=r_{\mathrm{rad}}^{\mathrm{sp}}+r_{\text {nrad, }}^{\mathrm{sp}}$ where $r_{\mathrm{f}}$ is the fluorescence decay rate and $r_{\text {nrad }}^{\text {sp }}$ is the nonradiative decay rate with plasmon. The fluorescence quantum efficiency $\eta(d)$ can be written as ${ }^{56}$

$$
\eta(d)=r_{\text {rad }}^{\text {sp }} / r_{\mathrm{f}}=g l(d, t=0) / r_{\mathrm{f}}
$$

where $d$ is the separation distance between the Au particle and dye. $I(d, t)$ is the intensity of measured fluorescence transient, and $g$ is the collection efficiency factor, which can be determined from R6G dye molecules since $\eta(d)=0.95$, $I(t=0)$, and $r_{\mathrm{f}}$ are experimentally determined values. From eq 7, we can obtain $r_{\text {rad }}^{\text {sp }}=g l(d, t=0)$. Therefore, we can deduce the nonradiative rate. In Figure $4 c$, the nonradiative rate $r_{\text {nrad }}^{\text {sp }}$ is plotted versus the spacer thickness, which increases by more than 3 orders of magnitude at $2.8 \mathrm{~nm}$ compared to pure R6G dye molecules $\left(1.1 \times 10^{7} \mathrm{~s}^{-1}\right)$; meanwhile, the quantum efficiency drops by more than 3 orders of magnitude. Therefore, the drastically enhanced nonradiative decay rate leads to a pronounced shortening of lifetime. The fluorescence excitation and decay can be shown as a simplified Jablonski diagram in Figure $4 \mathrm{~d}$. The electrons of the R6G molecule in ground states are excited into a higher state when they are excited by incident photons. The excitation transition rate, $\gamma_{\text {exc }}$ is proportional to the local field, which is enhanced when the dye molecule is near the plasmon structures. ${ }^{26}$ To make it simple, $\gamma_{0}$ and $\gamma_{\mathrm{sp}}$ are denoted to express the photon excitation rate of the R6G molecule and Au plasmon enhanced excitation rate, respectively, $\gamma_{\mathrm{exc}}=\gamma_{0}+\gamma_{\mathrm{sp}}$. On the other hand, the modified nonradiative decay rate due to plasmon, $r_{\text {nrad }}^{\text {new }}$ is induced when metallic nanoparticles approach the R6G molecule due to Förster energy transfer from exciton to lossy plasmon. Therefore, the nonradiative decay rate is accelerated, $r_{\text {nrad }}^{\mathrm{sp}}=r_{\text {nrad }}^{0}+r_{\text {nrad, }}^{\text {new }}$ where $r_{\text {nrad }}^{0}$ is the nonradiative decay rate without plasmon, which results in more energy transferred to the plasmon and quantum yield decreases. Previous reports in theory and experiment have proven that the increased nonradiative rate dominates the increased excitation rate leading to the quenching of the dye molecules when the silica spacer thickness is smaller than $8 \mathrm{~nm}^{26,39}$ In our experiments, the nonradiative rate is the fastest at $\sim 2.8 \mathrm{~nm}$, which justifies the minimum lifetime and quantum efficiency $(0.02 \%)$. Therefore, the rate of energy transfer from gain media to plasmon is the fastest at $\sim 2.8 \mathrm{~nm}$, which indicates that the most energy is transferred to the plasmon. Our results further reasonably confirm the strongest fluorophoreplasmon coupling resonance and the largest loss compensation at $\sim 2.8 \mathrm{~nm}$ spacer thickness. From the Förster dipole-dipole interaction model, it is found that the larger
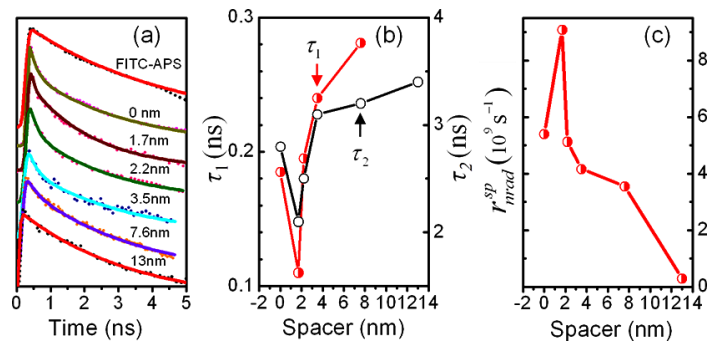

Figure 5. (a) Time-resolved fluorescence spectra of FITCAPS conjugate and Au/silica/FITC silica core-multishell structures. Silica spacer shells are $0,1.7,2.2,3.5,7.6$, and $13 \mathrm{~nm}$, respectively. The solid lines are fits to the data using an exponential decay function, as described in the text. (b) Lifetime as a function of the silica spacer thickness. (c) Nonradiative rate $\left(r_{\text {nrad }}^{\mathrm{sp}}\right)$ as a function of the silica spacer thickness; the unit is $10^{9} \mathrm{~s}^{-1}$.

the spectral overlap is, the faster the energy transfer is. It has been reported that both large spectral overlap and resonance between plasmon and emission of dye molecules can greatly improve energy transfer. ${ }^{21,49}$ In our experiments, the largest spectral overlap occurs at $\sim 2.8 \mathrm{~nm}$, manifesting a strong resonant R6G dye emission with a Au/silica core-shell nanostructure surface plasmon band. Therefore, we attribute the decrease of fluorescence lifetime to the spectral overlap and resonance between plasmon and R6G emission.

To further investigate the effect of spectral overlap and resonance on the energy transfer, we replace the R6G dyes by FITC dyes as the gain media to design $\mathrm{Au} /$ silica/FITC silica core-multishell nanostructures, and the middle silica shell spacers are $0,1.7 \pm 0.2,2.2 \pm 0.2$, $3.5 \pm 0.4,7.6 \pm 0.9$, and $13 \pm 0.9 \mathrm{~nm}$, respectively. The PL position of the FITC-APS conjugate is at $514 \mathrm{~nm}$ (Figure 2d), at the blue side of the plasmon band. Figure 5a shows the time-resolved PL spectra of the FITC-APS conjugate and the Au/silica/FITC silica coremultishell nanostructures, with each spacer thickness specified. For the FITC-APS conjugate, the timeresolved fluorescence intensity decay is fitted with a single-exponential function, giving rise to a time constant of $4.3 \pm 0.1 \mathrm{~ns}$, close to the previous report. ${ }^{59}$ However, for Au/silica/FITC silica core-multishell nanostructures, two time constants are observed, a shortliving lifetime $\tau_{1}$ and long-living lifetime $\tau_{2}$ when the silica spacer thickness is smaller than approximately $8 \mathrm{~nm}$. Figure $5 \mathrm{~b}$ shows the lifetime as a function of the spacer thickness. The fluorescence lifetimes $\tau_{1}$ are $185 \pm$ $4,110 \pm 11,195 \pm 8,240 \pm 22$, and $281 \pm 24$ ps and $\tau_{2}$ are $2.8 \pm 0.1,2.1 \pm 0.1,2.5 \pm 0.1,3.1 \pm 0.1$, and $3.2 \pm$ $0.1 \mathrm{~ns}$ for the silica shell spacer of $0,1.7,2.2,3.5$, and $7.6 \mathrm{~nm}$, respectively. Both time constants decrease to a minimum when the silica spacer is $1.7 \mathrm{~nm}$. We assign the two lifetimes to FITC dye molecules located close to and far away from the Au core, respectively. When the silica spacer is $13 \mathrm{~nm}$, there is only one long-living lifetime (3.4 $\pm 0.1 \mathrm{~ns}$ ), suggesting that the coupling between the plasmon and exciton of FITC becomes very weak. 
For the long-living lifetime $\tau_{2}$, the increase rate declines when the spacer is larger than $3.5 \mathrm{~nm}$, which further indicates that the plasmon-exciton coupling becomes weak as the spacer thickness is considerably large (e.g., $8 \mathrm{~nm}$ in this case). This also verifies that the long-living lifetime is from those dye molecules located far away from the Au core. Figure $4 \mathrm{c}$ shows the nonradiative rate as a function of the spacer thickness. The nonradiative rate $r_{\text {nrad }}^{\mathrm{sp}}$ increases by approximately 2 orders at $1.7 \mathrm{~nm}$ compared to the FITC-APS conjugate $\left(1.2 \times 10^{8} \mathrm{~s}^{-1}\right)$, where the quantum efficiency decreases more than 3 orders of magnitude. Compared to the results of R6G, we find that the nonradiative rate $r_{\text {nrad }}^{\text {sp }}$ increases to a maximum at a spacer thickness of $1.7 \mathrm{~nm}$ and then monotonously decreases with the increasing spacer thickness for FITC-doped plasmonic nanostructures. However, for R6G-doped plasmonic nanostructures, $r_{\text {nrad }}^{\text {sp }}$ first increases to achieve a maximum at $2.8 \mathrm{~nm}$, where the plasmon band $(551 \mathrm{~nm})$ is in resonance with the emission of R6G $(550 \mathrm{~nm})$ and the spectral overlap is the largest, and then decreases monotonously as the silica spacer thickness further increases. Therefore, the spectral overlap and the plasmonexciton resonance at short distances lead to a new nonradiative channel and accelerate the nonradiative decay rate, resulting in short-living lifetime and low quantum efficiency. Larger spectral overlap leads to a larger nonradiative energy transfer rate from the gain medium to the plasmon. We suggest that only when the plasmon band is in close resonance with the emission of fluorophores, and the distance between plasmon and the gain media is in a few nanometer proximity, a maximum nonradiative rate and the largest loss compensation can be readily achieved.

\section{CONCLUSION}

In summary, Au/silica core-shell and Au/silica/dyedoped silica core-multishell nanostructures have been synthesized with a tunable silica shell as a spacer with thickness ranging from $\sim 1.7$ to $108.4 \mathrm{~nm}$, where the plasmon band is red-shifted from 544 to $565 \mathrm{~nm}$. Such exquisite structures enable the systemic investigations of the energy transfer between active dye molecules and surface plasmon as a function of the spacer thickness. Our results show that the largest fluorescence quenching and plasmon loss compensation with R6G molecules can be achieved when the spacer is $\sim 2.8 \mathrm{~nm}$, where the spectral overlap between plasmon and R6G emission is maximized. The timeresolved fluorescence spectroscopy of the coremultishell system with dye embedded shows that the lifetime drops from $4.1 \mathrm{~ns}$ to $65 \mathrm{ps}$ and the nonradiative rate increases by more than 3 orders of magnitude at $\sim 2.8 \mathrm{~nm}$, resulting in the largest plasmon loss compensation from gain materials. As a comparison, the core-multishell nanostructures doped with FITC molecules, which exhibit much smaller spectral overlap with plasmon, show that the decay lifetime decreases to a minimum and the nonradiative rate increases to a maximum at $1.7 \mathrm{~nm}$. Our results suggest that the loss compensation is critically dependent on both the spectral overlap and the distance between plasmon and gain materials with nanometer accuracy in the sub-10 $\mathrm{nm}$ regime.

\section{METHODS}

Materials. (3-Aminopropyl)trimethoxysilane (APS), tetraethoxysilane (TEOS), chloroauric acid $\left(\mathrm{HAuCl}_{4} \cdot 3 \mathrm{H}_{2} \mathrm{O}\right)$, sodium citrate anhydrous, Rhodamine 6G (R6G), fluorescein isothiocyanate (FITC), 2-propanol (IPA, HLPC), ethanol (HLPC), sodium hydroxide $(\mathrm{NaOH})$, and ammonia were purchased from SigmaAldrich. Sodium silicate was purchased from Beijing Chemical Regent Company. Milli-Q water was used in all of the preparations.

Synthesis of Au/Silica Core-Shell Structures. We first synthesized Au NPs with a diameter of about $64 \mathrm{~nm}$ as cores by a standard sodium citrate reduction method. ${ }^{60}$ A freshly prepared aqueous solution of $1 \mathrm{mM}$ (3-aminopropyl)trimethoxysilane (APS) $(0.4 \mathrm{~mL})$ was added to $20 \mathrm{~mL}$ of as-prepared gold sol and $80 \mathrm{~mL}$ of Milli-Q water under vigorous magnetic stirring in 15 min, ensuring complete complexation of the amine groups with the gold surface. Then, $1.6 \mathrm{~mL}$ of $0.54 \mathrm{wt} \%$ sodium silicate solution and $2 \mathrm{~mL}$ of $0.1 \mathrm{M} \mathrm{NaOH}$ were added to the sol again under vigorous magnetic stirring. ${ }^{38}$ To accelerate the synthesis procedure and make the ultrathin silica shell, we elevated the reaction temperature from room temperature to $90{ }^{\circ} \mathrm{C}$. ${ }^{61}$ The thickness of thin silica shell can be tuned between 1 and $3 \mathrm{~nm}$ by controlling the reaction time. Au core/thin silica shell structures were obtained by centrifugation (9000 rpm, $5 \mathrm{~min}$ ) and redispersed in $6 \mathrm{~mL}$ of Milli-Q water. To increase the thickness of the silica shell, $0.36 \mathrm{~mL}$ of as-prepared Au/silica was added into $1.14 \mathrm{~mL}$ of water, $5 \mathrm{~mL}$ of IPA, and $0.125 \mathrm{~mL}$ of ammonia, followed by the addition of $75 \mu \mathrm{L}$ of tetraethoxysilane (TEOS,
$10 \mathrm{mM}$ ) and stirring for $24 \mathrm{~h}$. Au/silica core-shell nanostructures were centrifugated at $9000 \mathrm{rpm}$ for $5 \mathrm{~min}$ and redispersed in $1.5 \mathrm{~mL}$ of Milli-Q water. Silica shell thickness was then increased to $3.5 \mathrm{~nm}$. The thickness of the silica shell can further be tuned between 3.5 and $108.4 \mathrm{~nm}$ by increasing the amount of TEOS. On the basis of the Au/silica core-shell structures, we systematically investigated the distance dependence of energy transfer between the plasmon and R6G; $5.12 \times 10^{9} \mathrm{Au} / \mathrm{silica}$ core-shell particles were dispersed in $3 \mathrm{~mL}$ of DI water containing $2.83 \times 10^{-7} \mathrm{M} \mathrm{R6G}$, which were prepared freshly and kept in the dark before measurements.

Synthesis of Au/Silica/Dye-Doped Silica Core-Multishell Nanostructures. For Au/silica/R6G silica core-multilshell structures, $1.5 \mathrm{~mL}$ of obtained Au/silica core-shell nanostructures was added into $5 \mathrm{~mL}$ of IPA and $0.125 \mathrm{~mL}$ of ammonia, followed by the addition of $400 \mu \mathrm{L}$ of TEOS $(10 \mathrm{mM}$ ) twice within $4 \mathrm{~h}$ (at a interval of $2 \mathrm{~h}$ ) and $100 \mu \mathrm{L}$ of R6G solution ( $1 \mathrm{mM}$ ), stirring for $24 \mathrm{~h}$ in the dark. Au/silica/R6G silica core-multilshell structures can be obtained by centrifugation ( $6000 \mathrm{rpm}, 3 \mathrm{~min}$ ), washed five times by Milli-Q water to remove free R6G, redispersed in $0.2 \mathrm{~mL}$ of Milli-Q water, and kept in the dark before measurements.

For Au/silica/FITC silica core-multishell nanostructures, the experimental steps are the same except for using $20 \mu \mathrm{L}$ of FITCAPS conjugate instead of R6G solution, which was formed by stirring FITC $(6.2 \mathrm{mg})$ in $25 \mathrm{~mL}$ of ethanol containing $5.65 \mu \mathrm{L}$ of APS in the dark for $24 \mathrm{~h}^{62,63}$

Time-Resolved Fluorescence Intensity Decays. Excitation pulses were generated from an optical parametric amplifier (TOPAS, 
Light Conversion Ltd.) that was pumped by a $1 \mathrm{kHz}, 150 \mathrm{fs} \mathrm{Ti}$ : sapphire regenerative amplifier (Legend, Coherent, Inc.). The PL emission was collected in a standard backscattering geometry and dispersed by a 0.25 m DK240 spectrometer with a $150 \mathrm{~g} /$ $\mathrm{mm}$ grating. The PL signal was time-resolved using an Optronis Optoscope streak camera system which has an ultimate temporal resolution of $6 \mathrm{ps}$. Samples were loaded in cuvettes of $2 \mathrm{~mm}$ path length, and excitation wavelength was tuned to $480 \mathrm{~nm}$ for the Au/silica/R6G silica samples and to $490 \mathrm{~nm}$ for the Au/silica/FITC silica samples.

Characterization. TEM was performed on a JEOL1400 transmission electron microscope with an accelerating voltage of $100 \mathrm{kV}$. The spacer thickness was obtained by measurement and statistical analysis of many TEM images (>100 nanoparticles). UV/vis absorption spectra were recorded at room temperature using a Lambda 950 spectrophotometer. Room-temperature steady-state PL was recorded by Fluorolog with a $430 \mathrm{~W}$ Xe lamp, and the excitation wavelength is $480 \mathrm{~nm}$. In case R6G and FITC molecules were photobleached in our experimnts, the dye solution was freshly prepared. All samples were kept in the dark before measurements, which were finished within 5 min for each sample.

Conflict of Interest: The authors declare no competing financial interest.

Acknowledgment. Q.X. thanks the strong support from Singapore National Research Foundation through Singapore NRF fellowship grant (NRF-RF-2009-06) and Nanyang Technological University via start-up grant (M58110061) and New Initiative Fund (M58110100). H.V.D. and Q.X. gratefully acknowledge the substantial support from Singapore National Research Foundation through the Competitive Research Program (NRF-CRP-6-2010-2).

\section{REFERENCES AND NOTES}

1. Brongersma, M. L.; Shalaev, V. M. The Case for Plasmonics. Science 2010, 328, 440-441.

2. Zheludev, N. I.; Prosvirnin, S. L.; Papasimakis, N.; Fedotov, V. A. Lasing Spaser. Nat. Photonics 2008, 2, 351-354.

3. Wang, H.; Brandl, D. W.; Nordlander, P.; Halas, N. J. Plasmonic Nanostructures: Artificial Molecules. Acc. Chem. Res. 2007, 40, 53-62.

4. Luk'yanchuk, B.; Zheludev, N. I.; Maier, S. A.; Halas, N. J.; Nordlander, P.; Giessen, H.; Chong, C. T. The Fano Resonance in Plasmonic Nanostructures and Metamaterials. Nat. Mater. 2010, 9, 707-715.

5. Stockman, M. I. Spaser Action, Loss Compensation, and Stability in Plasmonic Systems with Gain. Phys. Rev. Lett. 2011, 106, 156802.

6. De Luca, A.; Grzelczak, M. P.; Pastoriza-Santos, I.; LizMarzán, L. M.; La Deda, M.; Striccoli, M.; Strangi, G. Dispersed and Encapsulated Gain Medium in Plasmonic Nanoparticles: A Multipronged Approach To Mitigate Optical Losses. ACS Nano 2011, 5, 5823-5829.

7. Liu, S.-Y.; Li, J.; Zhou, F.; Gan, L.; Li, Z.-Y. Efficient Surface Plasmon Amplification from Gain-Assisted Gold Nanorods. Opt. Lett. 2011, 36, 1296-1298.

8. Zheludev, N. I. The Road Ahead for Metamaterials. Science 2010, 328, 582-583.

9. Xiao, S.; Drachev, V. P.; Kildishev, A. V.; Ni, X.; Chettiar, U. K.; Yuan, H.-K.; Shalaev, V. M. Loss-Free and Active Optical Negative-Index Metamaterials. Nature 2010, 466, 735-738.

10. Pendry, J. B.; Maier, S. A. Comment on "Spaser Action, Loss Compensation, and Stability in Plasmonic Systems with Gain". Phys. Rev. Lett. 2011, 107, 259703.

11. Wuestner, S.; Pusch, A.; Tsakmakidis, K. L.; Hamm, J. M.; Hess, O. Comment on "Spaser Action, Loss Compensation, and Stability in Plasmonic Systems with Gain". Phys. Rev. Lett. 2011, 107, 259701

12. Wuestner, S.; Pusch, A.; Tsakmakidis, K. L.; Hamm, J. M.; Hess, O. Overcoming Losses with Gain in a Negative Refractive Index Metamaterial. Phys. Rev. Lett. 2010, 105, 127401.

13. Pusch, A.; Wuestner, S.; Hamm, J. M.; Tsakmakidis, K. L.; Hess, O. Coherent Amplification and Noise in Gain-Enhanced
Nanoplasmonic Metamaterials: A Maxwell-Bloch Langevin Approach. ACS Nano 2012, 6, 2420-2431.

14. Plum, E.; Fedotov, V. A.; Kuo, P.; Tsai, D. P.; Zheludev, N. I. Towards the Lasing Spaser: Controlling Metamaterial Optical Response with Semiconductor Quantum Dots. Opt. Express 2009, 17, 8548-8551.

15. Tanaka, K.; Plum, E.; Ou, J. Y.; Uchino, T.; Zheludev, N. I. Multifold Enhancement of Quantum Dot Luminescence in Plasmonic Metamaterials. Phys. Rev. Lett. 2010, 105, 227403.

16. Noginov, M. A.; Zhu, G.; Belgrave, A. M.; Bakker, R.; Shalaev, V. M.; Narimanov, E. E.; Stout, S.; Herz, E.; Suteewong, T.; Wiesner, U. Demonstration of a Spaser-Based Nanolaser. Nature 2009, 460, 1110-1112.

17. Stockman, M. I. Spasers Explained. Nat. Photonics 2008, 2, 327-329.

18. Das, S. R. The Spaser Nanolaser. IEEE Spectrum 2009, 46, 14-16.

19. Zhang, X.; Oulton, R. F.; Sorger, V. J.; Zentgraf, T.; Ma, R. M.; Gladden, C.; Dai, L.; Bartal, G. Plasmon Lasers at Deep Subwavelength Scale. Nature 2009, 461, 629-632.

20. Bardhan, R.; Grady, N. K.; Cole, J. R.; Joshi, A.; Halas, N. J. Fluorescence Enhancement by Au Nanostructures: Nanoshells and Nanorods. ACS Nano 2009, 3, 744-752.

21. Ginger, D. S.; Chen, Y.; Munechika, K. Dependence of Fluorescence Intensity on the Spectral Overlap between Fluorophores and Plasmon Resonant Single Silver Nanoparticles. Nano Lett. 2007, 7, 690-696.

22. Ming, T.; Zhao, L.; Chen, H.; Woo, K. C.; Wang, J.; Lin, H.-Q. Experimental Evidence of Plasmophores: Plasmon-Directed Polarized Emission from Gold Nanorod-Fluorophore Hybrid Nanostructures. Nano Lett. 2011, 11, 2296-2303.

23. Chen, H.; Ming, T.; Zhao, L.; Wang, F.; Sun, L.-D.; Wang, J.; Yan, C.-H. Plasmon-Molecule Interactions. Nano Today 2010, 5, 494-505.

24. Ginger, D. S.; Munechika, K.; Chen, Y. C.; Tillack, A. F.; Kulkarni, A. P.; Jen-La Plante, I.; Munro, A. M. Quantum Dot/Plasmonic Nanoparticle Metachromophores with Quantum Yields That Vary with Excitation Wavelength. Nano Lett. 2011, 11, 2725-2730.

25. Zhang, Q.; Shan, X. Y.; Feng, X.; Wang, C. X.; Wang, Q. Q.; Jia, J. F.; Xue, Q. K. Modulating Resonance Modes and Q Value of a CdS Nanowire Cavity by Single Ag Nanoparticles. Nano Lett. 2011, 11, 4270-4274.

26. Novotny, L.; Anger, P.; Bharadwaj, P. Enhancement and Quenching of Single-Molecule Fluorescence. Phys. Rev. Lett. 2006, 96, 113002.

27. Aslan, K.; Wu, M.; Lakowicz, J. R.; Geddes, C. D. Fluorescent Core-Shell Ag@SiO 2 Nanocomposites for MetalEnhanced Fluorescence and Single Nanoparticle Sensing Platforms. J. Am. Chem. Soc. 2007, 129, 1524-1525.

28. Kulakovich, O.; Strekal, N.; Yaroshevich, A.; Maskevich, S.; Gaponenko, S.; Nabiev, I.; Woggon, U.; Artemyev, M. Enhanced Luminescence of CdSe Quantum Dots on Gold Colloids. Nano Lett. 2002, 2, 1449-1452.

29. Jennings, T. L.; Schlatterer, J. C.; Singh, M. P.; Greenbaum, N. L.; Strouse, G. F. NSET Molecular Beacon Analysis of Hammerhead RNA Substrate Binding and Catalysis. Nano Lett. 2006, 6, 1318-1324.

30. Pons, T.; Medintz, I. L.; Sapsford, K. E.; Higashiya, S.; Grimes, A. F.; English, D. S.; Mattoussi, H. On the Quenching of Semiconductor Quantum Dot Photoluminescence by Proximal Gold Nanoparticles. Nano Lett. 2007, 7, 3157-3164.

31. Tovmachenko, O. G.; Graf, C.; van den Heuvel, D. J.; van Blaaderen, A.; Gerritsen, H. C. Fluorescence Enhancement by Metal-Core/Silica-Shell Nanoparticles. Adv. Mater. 2006, 18, 91-95.

32. Kulakovich, O.; Strekal, N.; Yaroshevich, A.; Maskevich, S.; Gaponenko, S.; Nabiev, I.; Woggon, U.; Artemyev, M. Enhanced Luminescence of CdSe Quantum Dots on Gold Colloids. Nano Lett. 2002, 2, 1449-1452.

33. Schatz, G. C.; Kelly, K. L.; Coronado, E.; Zhao, L. L. The Optical Properties of Metal Nanoparticles: The linfluence of Size, Shape, and Dielectric Environment. J. Phys. Chem. B 2003, 107, 668-677. 
34. Link, S.; El-Sayed, M. A. Spectral Properties and Relaxation Dynamics of Surface Plasmon Electronic Oscillations in Gold and Silver Nanodots and Nanorods. J. Phys. Chem. B 1999, 103, 8410-8426.

35. Mulvaney, P.; Ung, T.; Liz-Marzan, L. M. Optical Properties of Thin Films of Au@SiO 2 Particles. J. Phys. Chem. B 2001, $105,3441-3452$.

36. Mulvaney, P. Surface Plasmon Spectroscopy of Nanosized Metal Particles. Langmuir 1996, 12, 788-800.

37. Mulvaney, P.; Liz-Marzan, L. M.; Giersig, M.; Ung, T. Silica Encapsulation of Quantum Dots and Metal Clusters. J. Mater. Chem. 2000, 10, 1259-1270.

38. Liz-Marzan, L. M.; Giersig, M.; Mulvaney, P. Synthesis of Nanosized Gold-Silica Core-Shell Particles. Langmuir 1996, 12, 4329-4335.

39. Schneider, G.; Decher, G.; Nerambourg, N.; Praho, R.; Werts, M. H. V.; Blanchard-Desce, M. Distance-Dependent Fluorescence Quenching on Gold Nanoparticles Ensheathed with Layer-by-Layer Assembled Polyelectrolytes. Nano Lett. 2006, 6, 530-536.

40. Zin, M. T.; Leong, K.; Wong, N. Y.; Ma, H.; Sarikaya, M.; Jen, A. K. Y. Surface-Plasmon-Enhanced Fluorescence from Periodic Quantum Dot Arrays through Distance Control Using Biomolecular Linkers. Nanotechnology 2009, 20, 015305.

41. Ray, K.; Badugu, R.; Lakowicz, J. R. Distance-Dependent Metal-Enhanced Fluorescence from Langmuir-Blodgett Monolayers of Alkyl-NBD Derivatives on Silver Island Films. Langmuir 2006, 22, 8374-8378.

42. Dulkeith, E.; Ringler, M.; Klar, T. A.; Feldmann, J.; Muñoz Javier, A.; Parak, W. J. Gold Nanoparticles Quench Fluorescence by Phase Induced Radiative Rate Suppression. Nano Lett. 2005, 5, 585-589.

43. Gersten, J.; Nitzan, A. Spectroscopic Properties of Molecules Interacting with Small Dielectric Particles. J. Chem. Phys. 1981, 75, 1139-1152.

44. Carminati, R.; Greffet, J. J.; Henkel, C.; Vigoureux, J. M. Radiative and Non-radiative Decay of a Single Molecule Close to a Metallic Nanoparticle. Opt. Commun. 2006, 261, 368-375.

45. Lakowicz, J. R. Principles of Fluorescence Spectroscopy, 3rd ed.; Springer: New York, 2006; pp 443-472.

46. Kubin, R. F.; Fletcher, A. N. Fluorescence Quantum Yields of Some Rhodamine Dyes. J. Lumin. 1982, 27, 455-462.

47. Shimizu, K. T.; Woo, W. K.; Fisher, B. R.; Eisler, H. J.; Bawendi, M. G. Surface-Enhanced Emission from Single Semiconductor Nanocrystals. Phys. Rev. Lett. 2002, 89, 117401.

48. Wang, Y. K.; Yang, T. Y.; Tuominen, M. T.; Achermann, M. Radiative Rate Enhancements in Ensembles of Hybrid Metal-Semiconductor Nanostructures. Phys. Rev. Lett. 2009, 102, 163001.

49. Tam, F.; Goodrich, G. P.; Johnson, B. R.; Halas, N. J. Plasmonic Enhancement of Molecular Fluorescence. Nano Lett. 2007, 7, 496-501.

50. Munechika, K.; Chen, Y.; Tillack, A. F.; Kulkarni, A. P.; Plante, I. J. L.; Munro, A. M.; Ginger, D. S. Spectral Control of Plasmonic Emission Enhancement from Quantum Dots near Single Silver Nanoprisms. Nano Lett. 2010, 10, 2598-2603.

51. Ma, R.-M.; Oulton, R. F.; Sorger, V. J.; Bartal, G.; Zhang, X. Room-Temperature Sub-Diffraction-Limited Plasmon Laser by Total Internal Reflection. Nat. Mater. 2011, 10, 110113.

52. Kühn, S.; Håkanson, U.; Rogobete, L.; Sandoghdar, V. Enhancement of Single-Molecule Fluorescence Using a Gold Nanoparticle as an Optical Nanoantenna. Phys. Rev. Lett. 2006, 97, 017402.

53. Magde, D.; Wong, R.; Seybold, P. G. Fluorescence Quantum Yields and Their Relation to Lifetimes of Rhodamine $6 \mathrm{G}$ and Fluorescein in Nine Solvents: Improved Absolute Standards for Quantum Yields. Photochem. Photobiol. 2002, 75, 327-334.

54. Xu, X. L.; Zhao, Y. Y.; Sie, E. J.; Lu, Y. H.; Liu, B.; Ekahana, S. A.; Ju, X.; Jiang, Q. K.; Wang, J. B.; Sun, H. D.; et al. Dynamics of Bound Exciton Complexes in CdS Nanobelts. ACS Nano 2011, 5, 3660-3669.
55. Zhang, Y. X.; Mandeng, L. N.; Bondre, N.; Dragan, A.; Geddes, C. D. Metal-Enhanced Fluorescence from Silver$\mathrm{SiO}_{2}-$ Silver Nanoburger Structures. Langmuir 2010, 26, 12371-12376.

56. Dulkeith, E.; Morteani, A. C.; Niedereichholz, T.; Klar, T. A.; Feldmann, J.; Levi, S. A.; van Veggel, F. C. J. M.; Reinhoudt, D. N.; Möller, M.; Gittins, D. I. Fluorescence Quenching of Dye Molecules near Gold Nanoparticles: Radiative and Nonradiative Effects. Phys. Rev. Lett. 2002, 89, 203002.

57. Cannone, F.; Chirico, G.; Bizzarri, A. R.; Cannistraro, S. Quenching and Blinking of Fluorescence of a Single Dye Molecule Bound to Gold Nanoparticles. J. Phys. Chem. B 2006, 110, 16491-16498.

58. Lim, T. S.; Fu, C. C.; Lee, K. C.; Lee, H. Y.; Chen, K.; Cheng, W. F.; Pai, W. W.; Chang, H. C.; Fann, W. Fluorescence Enhancement and Lifetime Modification of Single Nanodiamonds near a Nanocrystalline Silver Surface. Phys. Chem. Chem. Phys. 2009, 11, 1508-1514.

59. Santra, S.; Liesenfeld, B.; Bertolino, C.; Dutta, D.; Cao, Z. H.; Tan, W. H.; Moudgil, B. M.; Mericle, R. A. Fluorescence Lifetime Measurements To Determine the Core-Shell Nanostructure of FITC-Doped Silica Nanoparticles: An Optical Approach To Evaluate Nanoparticle Photostability. J. Lumin. 2006, 117, 75-82.

60. Liu, S. H.; Han, M. Y. Synthesis, Functionalization, and Bioconjugation of Monodisperse, Silica-Coated Gold Nanoparticles: Robust Bioprobes. Adv. Funct. Mater. 2005, 15, 961-967.

61. Li, J. F.; Huang, Y. F.; Ding, Y.; Yang, Z. L.; Li, S. B.; Zhou, X. S.; Fan, F. R.; Zhang, W.; Zhou, Z. Y.; Wu, D. Y.; et al. ShellIsolated Nanoparticle-Enhanced Raman Spectroscopy. Nature 2010, 464, 392-395.

62. Chen, D.; Li, L. L.; Tang, F. Q.; Qi, S. O. Facile and Scalable Synthesis of Tailored Silica "Nanorattle" Structures. Adv. Mater. 2009, 21, 3804-3807.

63. Peng, B.; Tang, F. Q.; Chen, D.; Ren, M. L.; Meng, X. W.; Ren, J. Preparation of $\mathrm{PS} / \mathrm{TiO}_{2} / \mathrm{UF}$ Multilayer Core-Shell Hybrid Microspheres with High Stability. J. Colloid Interface Sci. 2009, 329, 62-66. 